\title{
Time synchronization method of Network Testing system by Standard Wave
}

\author{
Jongkuk Lee, Young-Seo Jeong, Ki-Dong Nam \\ ETRI(Electronics and Telecommunications Research Institute), Korea \\ raphael@etri.re.kr,jys847@etri.re.kr, kdnam@etri.re.kr
}

\begin{abstract}
The time synchronization is very important for network testing that is at a long distance. For time synchronization, two different network testing apparatuses uses a network time protocol (NTP) or a geographic position system (GPS).

For using GPS with less timing error, an expensive GPS antenna needs to be installed outside the door. NTP is through the network. The network itself causes a time delay and the error in the time delay of the network occurs. So, NTP has not been used to measure a high speed network.

This paper has been made in an effort to provide a network testing system using a Standard Wave to facilitate time synchronization with at least two network testing apparatuses, and an operating method thereof.
\end{abstract}

The Standard Wave has extremely little time error. It helps to perform accurate testing.

Keywords - Standard Wave, Time Synchronization, Testing

\section{INTRODUCTION}

When a general network testing apparatus (packet generator $\&$ analyser) performs network testing, only using one tester has not timing problem. But, two or more testers have to use between one and the other positions between long distance, have timing problem. Because two testers must have a same clock. If two testers have different clocks, testers are not able to measure delay, jitter, etc.

It is a timing synchronization problem. In this time, for solving timing synchronization problem, we use a network time protocol (NTP) or a geographic position system (GPS).

For using GPS with less timing error, an expensive GPS antenna needs to be installed outside the door. Since the NTP performs the time synchronization through the network, the network itself causes a time delay and the error in the time delay of the network occurs, such that the NTP has not been used to measure a high speed network.

In this paper, we suppose method of network testing system by Standard Wave.

\section{RELATED WORKS}

The Standard Wave extremely correct frequency electronic wave. It has error tolerance is $\pm 2 \times 10^{-8} \mathrm{~ns}$ (Nano second) and is standard of electronic wave frequency. It has correct time data or wave signal. [1]
A radio clock is a clock that is synchronized by a time code bit stream transmitted by a radio transmitter connected to a time standard such as an atomic clock. Such a clock may be synchronized to the time sent by a single transmitter, such as many national or regional time transmitters, or may use multiple transmitters, like the Global Positioning System. Such systems may be used to automatically set clocks or for any purpose where accurate time is needed [2].

Time signal transmission is using by Japan, Russia, US, UK, China, Switzerland, Germany, France, Canada, South Korea, Argentina, Venezuela, Brazil, India and Taiwan.

Its Frequency are 40, 50, 60, 66.66, 68.5, 77.5, 100, 162 $\mathrm{kHz}$ and 2.5, 3.33, 4.996, 5, 7.85, 9.996, 10, 11, 14.67, 14.996, 15, $20 \mathrm{MHZ}$.

\section{A. Current Time synchronization}

Different locations to measure the network delay, the time should be synchronized. Of course, divided in half by measuring the round-trip time from one place to another, but it must be the same transmission time of send and receive.

If you want to measure exactly one-way time, you must be synchronized two place network tester's time data.

The Time Synchronization Methods of network is two. One is NTP (Network Time Protocol).

The Network Time Protocol (NTP) is widely used to synchronize computer clocks in the Internet. An NTP implementation operates as a primary server, secondary server, or client. A primary server is synchronized to a reference clock directly traceable to UTC (e.g., GPS, Galileo, etc.). A client synchronizes to one or more upstream servers, but does not provide synchronization to dependent clients. A secondary server has one or more upstream servers and one or more downstream servers or clients. [3]

A time difference of less than $128 \mathrm{~ms}$ between server and client is required to maintain NTP synchronization. The typical accuracy on the Internet ranges from about $5 \mathrm{~ms}$ to $100 \mathrm{~ms}$, possibly varying with network delays. A recent survey [4] suggests that $90 \%$ of the NTP servers have network delays below $100 \mathrm{~ms}$, and about $99 \%$ are synchronized within one second to the synchronization peer. So, Asymmetric routes and network congestion can cause errors of 100ms or more. [5]

The other is GPS (Global Positioning System).

It is a space-based satellite navigation system that provides location and time information in all weather conditions, 
anywhere on or near the Earth where there is an unobstructed line of sight to four or more GPS satellites. The system provides critical capabilities to military, civil and commercial users around the world. It is maintained by the United States government and is freely accessible to anyone with a GPS receiver.

The GPS project was developed in 1973 to overcome the limitations of previous navigation systems, integrating ideas from several predecessors, including a number of classified engineering design studies from the 1960s. GPS was created and realized by the U.S. Department of Defence (DoD) and was originally run with 24 satellites. It became fully operational in 1994. Bradford Parkinson, Roger L. Easton, and Ivan A. Getting are credited with inventing it.

Advances in technology and new demands on the existing system have now led to efforts to modernize the GPS system and implement the next generation of GPS III satellites and Next Generation Operational Control System (OCX). [7]

But It has time error, about 1 10ns. Time error is due to ionization layer error, astronomical ephemeris error, satellite time clock error, wave propagation path error, troposphere error and numerical error. [8]

In [6], Mills attributes most of the phase error seen when synchronizing an NTP client workstation to a GPS receiver on the same $\operatorname{LAN}\left(500 \mu_{\mathrm{sec}} \sim 2000 \mu_{\mathrm{sec}}\right.$ in his 1994 study) to these factors -Ethernet jitter and collisions.

GPS system need that antenna is must be outdoor. So, GPS network tester is closely window or needs long cable from GPS.

\section{STANDARD WAVE}

In this paper, we suggest method of Standard Wave. Standard Wave is low frequency wave. It is not must be outdoor. And it doesn't need long cable. Just, it needs a radio antenna.

NTP has the Network Delay. Network delay is not consistent. If network tester uses NTP for time synchronization, time error correct is impossible. And time error is $5 \sim 100 \mathrm{~ms}$. Even, Network time delay is smaller than NTP server delay time.

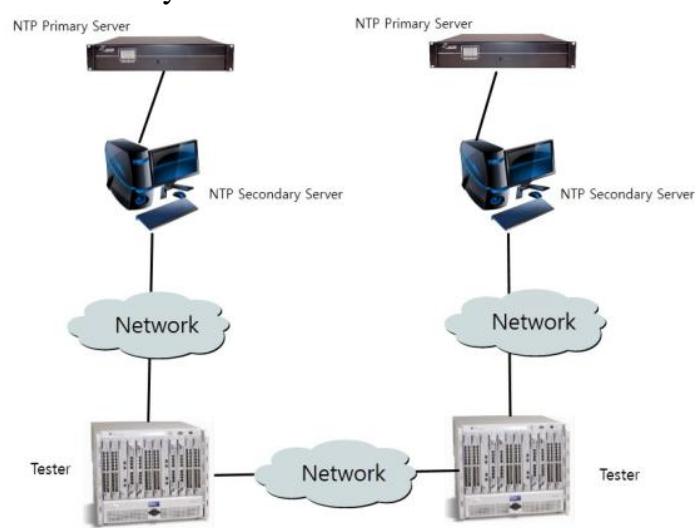

Figure 1. Network Test by NTP
GPS is very exact time synchronization method. But, it is very long distance. So, it has ionization layer error, astronomical ephemeris error, satellite time clock error, wave propagation path error, troposphere error and numerical error.

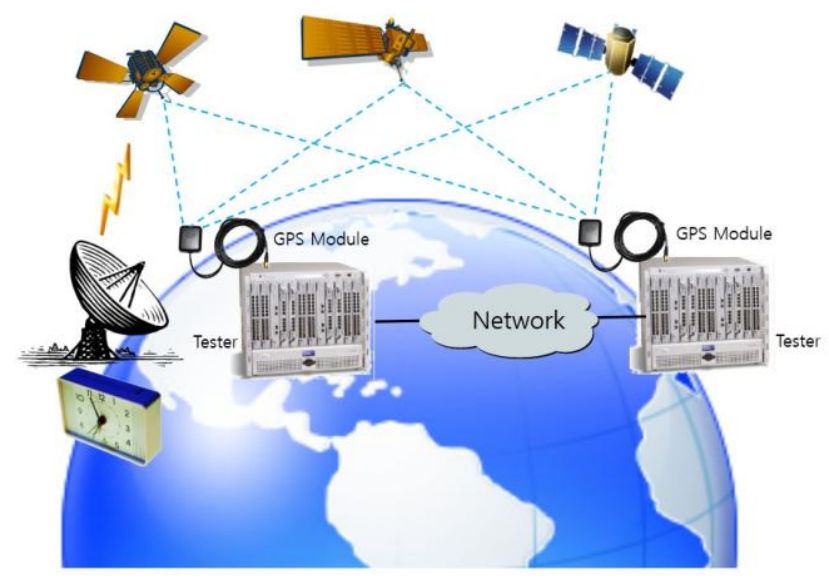

Figure 2. Network Test by GPS

Standard Wave is most exact time synchronization method. It has receiver device error and numerical error. So Standard Wave time is very small.

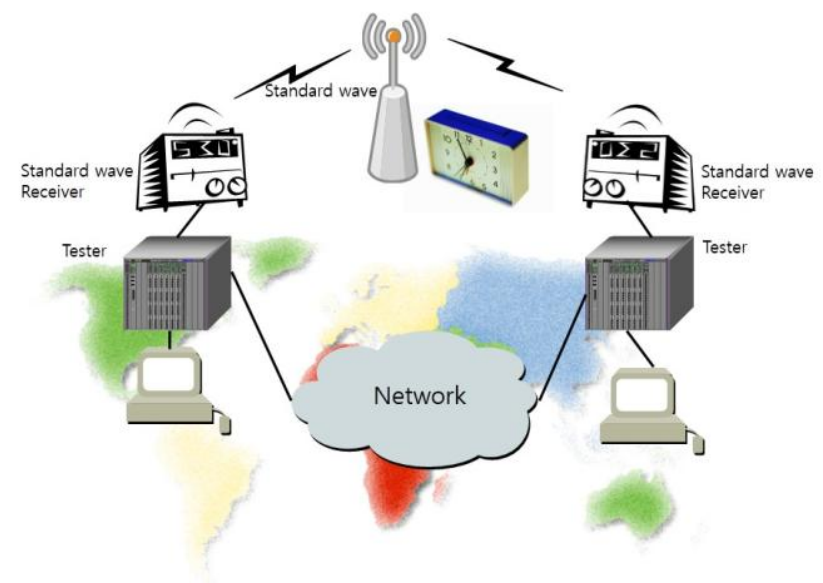

Figure 3. Network Test by Standard Wave

TABLE 1. TIME ERROR OF NETWORK SYSTEM

\begin{tabular}{|c|c|c|}
\hline \multicolumn{3}{|c|}{ Time error } \\
\hline Standard Wave & NTP & GPS \\
\hline $\pm 2 \times 10^{-8} \mathrm{~ns}$ & $\pm 5 \sim 100 \mathrm{~ms}$ & $\pm 1 \mathrm{~ns}$ \\
\hline
\end{tabular}


In this paper, we suggest Standard Wave method and algorithm.

\section{A. Standard Wave Time Synchronization Process}

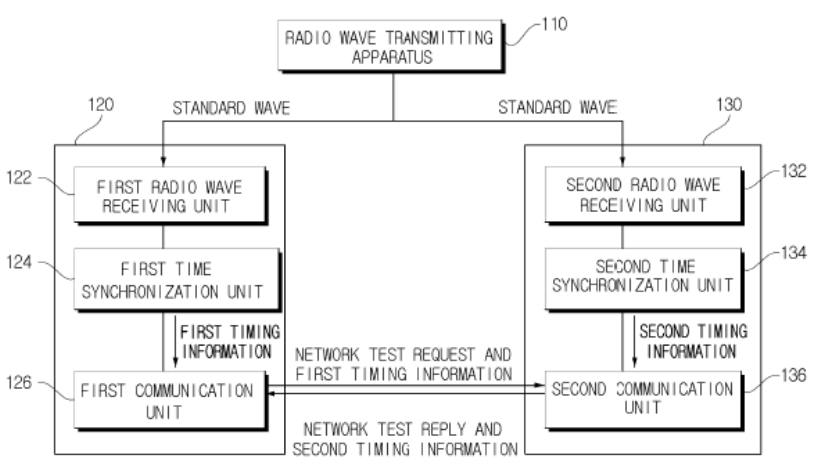

Figure 4. Standard Wave Time Synchronization Process

In Figure 4, The first network testing apparatus 120 may include a first radio wave receiving unit 122 which receives the Standard Wave, a first time synchronization unit 124 which performs time synchronization based on the timing data included in the Standard Wave and generates first timing information, and a first communication unit 126 which transmits a network test request and the first timing information to the second network testing apparatus 130 and receives a network test reply and second timing information from the second network testing apparatus 130 .

The second network testing apparatus 130 may include a second radio wave receiving unit 132 which receives the Standard Wave, a second time synchronization unit 134 which performs time synchronization based on the timing data included in the Standard Wave and generates the second timing information, and a second communication unit 136 which transmits the network test reply and the second timing information to the first network testing apparatus 130 when the network test request and the first timing information is transmitted from the first network testing apparatus 130 .

The exemplary embodiment of this paper illustrates and describes that the first and second network testing apparatuses 120 and 130 have the same configuration; however, the first and second network testing apparatuses 120 and 130 may have different configurations. Accordingly, the first and second network testing apparatuses 120 and 130 may not include at least one of the first and second communication units 126 and 136 , and therefore the exemplary embodiment of this paper is not limited thereto. Herein, each of the first and second time synchronization units 124 and 134 may perform time synchronization based on the timing data included in the standard signal to generate the first and second timing information.

That is, each of the first and second time synchronization units 124 and 134 performs time synchronization based on the timing data included in the standard signal to considerably reduce the timing error to several ns, thereby reducing the synchronization error between the first and second network testing apparatuses 120 and 130. Each of the first and second communication units 126 and 136 may perform wired and wireless communication with each other and may transmit and receive the network test request, the network test reply, and the like, through communication with each other.

The exemplary embodiment of this paper describes the first network testing apparatus 120, and the second network testing apparatus 130 may be identically operated with the first network testing apparatus 120 , but the exemplary embodiment of this paper is not limited thereto.

\section{B. Standard Wave Time Synchronization Flow Chart}

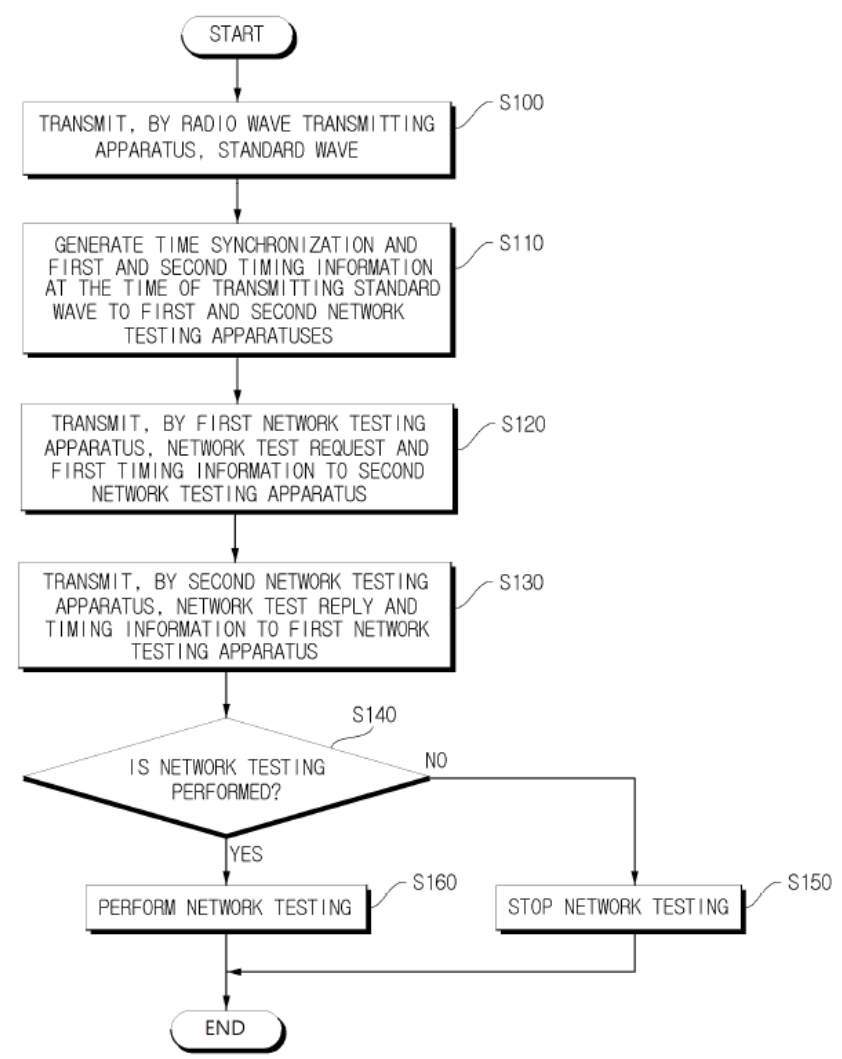

Figure 5. Standard Wave Time Synchronization Flow Chart

Figure 5. is a flow chart illustrating an operating method of a network testing system according to an exemplary embodiment of this paper.

Referring to Figure 5, the network testing system includes transmitting, by the radio wave transmitting apparatus, the Standard Wave including the timing data (S100) and when the first and second network testing apparatuses transmit the Standard Wave, performing, by the first and second network testing apparatuses, the time synchronization based on the 
timing data and generating the first and second timing information (S110).

That is, the radio wave transmitting apparatus transmits the Standard Wave including the timing data to each of the first and second network testing apparatuses. The first and second network testing apparatuses perform time synchronization based on the timing data at the time of receiving the Standard Wave and generate the first and second timing information regarding whether the time is synchronized.

The first network testing apparatus transmits the network test request and the first timing information to the second network testing apparatus (S120) and the second network testing apparatus transmits the network test reply corresponding to the network test request and the second timing information (S130).

That is, the first network testing apparatus transmits the network test request and the first timing information to the second network testing apparatus for network time synchronization with the second network testing apparatus at a remote place.

The second network testing apparatus transmits the network test reply and the second timing information regarding whether to accept the testing to the first network testing apparatus, based on the network test request transmitted from the first network testing apparatus.

The first network testing apparatus determines whether network testing is accepted and whether the time synchronization of the second network testing apparatus is performed, based on the network test reply and the second timing information transmitted from the second network testing apparatus (S140).

That is, the first network testing apparatus may determine whether the network testing is performed based on the second network test reply transmitted from the second network testing apparatus and may determine whether the time synchronization of the second network testing apparatus is performed according to the timing data based on the second timing information.

As the determination result in step (S140), when the second network testing apparatus rejects network testing and does not perform time synchronization, the first network testing apparatus stops the network testing along with the second network testing apparatus (S150) or when the second network testing apparatus performs the network testing and completes the time synchronization, the first network testing apparatus progresses the network testing along with the second network testing apparatus (S160).

\section{IV.CONCLUSIONS}

The Standard Wave is useful for time synchronization. It is working indoor and outdoor. It is very cheap. And, it is very exact time data. So, it is able to use for time synchronization. For the future works, we development this module at future.

\section{REFERENCES}

[1] Electronic terminology dictionary, Byong-Ho Kim, Il Jin Co., Sep. 2010.

[2] "Radio Clock", Wikipedia : http://en.wikipedia.org/wiki/Radio_clock, Oct.2013

[3] D.Mills, U.Delaware, etc., RFC 5905, "Network Time Protocol Version 4: Protocol and Algorithms Specification", IETF, Jun. 2010.

[4] "Executive Summary: Computer Network Time Synchronization". "http://www.ntp.org/ntpfaq/NTP-s-def.htm\#FTN.FTN-NTPSURVEY99", Retrieved 2011-11-21.

[5] "NTP FAQ"." http://www.ntp.org/ntpfaq/NTP-s-algo.htm", The NTP Project. Retrieved 2011-08-27.

[6] D. L. Mills. Precision synchronization of cmputer network clocks. ACM Computer Communication Review, 24(2):28-43, April 1994.

[7] "Global Positioning System", Wikipedia: http://en.wikipedia.org/wiki/Global_Positioning_Systrem, Oct. 26. 2013

[8] “GPS”, Wikipedia Korea: http://ko.wikipedia.org/wiki/GPS, Oct. 2013. 